\title{
Erratum to: Associations between adult attachment and: oral health-related quality of life, oral health behaviour, and self-rated oral health
}

\author{
Pamela Meredith $^{1} \cdot$ Jenny Strong $^{1} \cdot$ Pauline Ford $^{2} \cdot$ Grace Branjerdporn $^{1}$
}

Published online: 25 November 2016

(c) Springer International Publishing Switzerland 2016

\section{Erratum to: Qual Life Res (2016) 25:423-433 \\ DOI 10.1007/s11136-015-1089-1}

Unfortunately, in the original publication of the article, the variables of "Dental Self-Care" and "Dental Visiting" have been inadvertently swapped in Table 4 . The correct version of the table is provided in this erratum (Table 4).

The online version of the original article can be found under doi:10.1007/s11136-015-1089-1.

Pamela Meredith

p.meredith@uq.edu.au

1 Occupational Therapy, School of Health and Rehabilitation Sciences, The University of Queensland, Brisbane, QLD 4072, Australia

2 School of Dentistry, The University of Queensland, Brisbane, QLD, Australia 
Table 4 Regression coefficients from multivariate regression models of attachment anxiety and attachment avoidance predicting OHIP-14, m-DNS, and self-rated oral health, when controlling for demographic characteristics and oral health behaviours

\begin{tabular}{|c|c|c|c|c|}
\hline & \multicolumn{2}{|c|}{ Attachment anxiety } & \multicolumn{2}{|c|}{ Attachment avoidance } \\
\hline & $\beta$ & Model $R^{2}(\operatorname{adj})$ & $\beta$ & Model $R^{2}(\operatorname{adj})$ \\
\hline \multicolumn{5}{|l|}{ OHIP-14 } \\
\hline Overall score (OHIP-14S) & $0.17 * *$ & $0.21 * * *$ & $0.15 * *$ & $0.21 * * *$ \\
\hline Functional Limitation & $0.13 *$ & $0.09 * * *$ & $0.24 * * *$ & $0.13 * * *$ \\
\hline Physical Pain & 0.06 & $0.14 * * *$ & 0.09 & $0.14 * * *$ \\
\hline Psychological Discomfort & $0.22 * * *$ & $0.14 * * *$ & $0.17 * *$ & $0.12 * * *$ \\
\hline Physical Disability & 0.01 & $0.15 * * *$ & 0.02 & $0.15 * * *$ \\
\hline Psychological Disability & $0.18 * *$ & $0.18 * * *$ & $0.11^{*}$ & $0.16 * * *$ \\
\hline Social Disability & $0.16^{* *}$ & $0.15 * * *$ & 0.08 & $0.13 * * *$ \\
\hline Handicap & $0.19 * *$ & $0.16^{* * *}$ & $0.15^{* *}$ & $0.14 * * *$ \\
\hline \multicolumn{5}{|l|}{ Modified Dental Neglect scale } \\
\hline Dental Visiting $^{\mathrm{b}}$ & $-0.15^{*}$ & $0.06 * * *$ & $-0.12 *$ & $0.05^{* *}$ \\
\hline Dental Self-Care $^{c}$ & -0.05 & $0.07 * * *$ & -0.06 & $0.07 * * *$ \\
\hline Self-rated oral health ${ }^{\mathrm{a}}$ & -0.06 & $0.19 * * *$ & $-0.25 * * *$ & $0.25 * * *$ \\
\hline
\end{tabular}

$* p<0.05 ; * * p<0.01, * * * p<0.001$

${ }^{a}$ Covariates included gender, tooth brushing frequency, flossing frequency, and reason for dental visit

b Covariates included gender, tooth brushing frequency, and flossing frequency

${ }^{c}$ Covariates included gender and reason for dental visit 